\title{
Evaluación de la mejora del proceso de enseñanza-aprendizaje mediante la integración de objetos de aprendizaje reutilizables en un curso abierto $\mathrm{OCW}$
}

\author{
Ignacio Zabalza a , Begoña Peña, Eva María Llera, Sergio Usón, Amaya Martínez, Luis \\ Miguel Romeo \\ Universidad de Zaragoza - Departamento de Ingeniería Mecánica, 50018 Zaragoza, España. ${ }^{a}$ e-mail: \\ izabal@unizar.es
}

\begin{abstract}
En un contexto de Recursos Educativos Abiertos (REA), el concepto de Objetos de Aprendizaje Reutilizables (OAR), en formato digital y de carácter independiente y autónomo, resulta de gran interés para su uso en contextos educativos múltiples como los cursos abiertos (OCW, por sus siglas en inglés).

El objetivo de este trabajo es presentar los principales resultados obtenidos hasta la fecha en un proyecto de innovación docente financiado por la Universidad de Zaragoza durante los cursos académicos 2015-16 y 2016-17 (Ref. PIIDUZ_15_079, $16 \_165$ y 16_032). En este proyecto, se han creado 25 OARs en forma de videos docentes bajo licencias Creative Commons para mejorar los resultados de aprendizaje de cinco asignaturas en el campo de la Termodinámica y la Ingeniería Térmica, que actualmente se imparten en tres titulaciones distintas. Para evaluar la mejora del proceso de enseñanzaaprendizaje obtenida mediante los vídeos, se han preparado y lanzado diferentes cuestionarios para los estudiantes.

Hasta el momento, los resultados muestran que la mayoría de los estudiantes están satisfechos con esta experiencia de innovación. En este sentido, consideran que el uso de videos docentes ha mejorado su proceso de aprendizaje, aumentando su motivación para estudiar y ayudándoles a entender los conceptos más complejos.
\end{abstract}

Keywords: $O A R, O C W, R E A$, vídeos docentes, innovación docente.

\section{Introducción}

Un buen número de Universidades englobadas dentro del Consorcio de Educación Abierta (Open Education Consortium), entre las que se incluye la Universidad de Zaragoza (España), están promoviendo el acceso abierto al conocimiento a través de portales de acceso público que incorporan un conjunto de cursos abiertos OpenCourseWare (OCW), poniéndolos así a disposición de toda la comunidad de profesores, estudiantes o cualquier persona que desee aprender sobre una determinada materia. 
Uno de los principales desafíos a la hora de diseñar un OCW es cómo producir, gestionar y publicar contenidos de calidad, que puedan ser revisados y actualizados de un modo eficiente. Con este fin, surge el concepto de los Objetos de Aprendizaje Reutilizables (OAR) que constituyen elementos de aprendizaje autónomos con posibilidad de ser reutilizados en distintos contextos educativos. Por lo general, los OAR utilizan elementos multimedia accesibles por los estudiantes en cualquier momento y en cualquier lugar. De este modo, se proporciona al alumnado una experiencia de aprendizaje visual e interactiva, que puede incluir diversos elementos de autoevaluación, facilitando así el aprendizaje autónomo (Rehak y Mason, 2003).

Diversos estudios sugieren que los OAR constituyen un buen complemento a la enseñanza tradicional, contribuyendo a mejorar los resultados de la evaluación, aumentando la eficacia del aprendizaje (Windle et al., 2011; Zabalza et al., 2017), pudiendo utilizarse también en el modelo de clase invertida (Peña et al., 2017).

Los OAR se plantean generalmente como una respuesta a una necesidad de aprendizaje, por lo que después de su implementación es importante analizar el grado en el que se ha satisfecho dicha necesidad (Kay y Knaack, 2007). Sin embargo, los criterios de calidad comúnmente evaluados actualmente siguen siendo bastantes diferentes (Leacock y Nesbit, 2007; Singh y Bernard, 2016), por lo que se hace necesaria una sistematización de conceptos y criterios para la evaluación de los OAR (Pinto, Gómez-Camarero y FernándezRamos, 2012).

En este trabajo se presenta la metodología seguida y los principales resultados obtenidos hasta la fecha en un proyecto de innovación docente en que se ha hecho una evaluación de la mejora del proceso de enseñanza-aprendizaje mediante el uso vídeos docentes como objetos de aprendizaje reutilizables integrables dentro de un curso OCW. Este proyecto ha sido financiado por la Universidad de Zaragoza durante los cursos académicos 2015-16 y 2016-17. En concreto, los objetivos específicos del proyecto de innovación son:

- Aprovechar las oportunidades que ofrecen las nuevas tecnologías del aprendizaje y del conocimiento para mejorar la calidad docente y el proceso de enseñanzaaprendizaje en asignaturas del ámbito de la termodinámica y la ingeniería térmica, que actualmente se imparten en la Escuela de Ingeniería y Arquitectura de la Universidad de Zaragoza.

- Actualizar y mejorar los videos didácticos producidos en el curso académico anterior, a partir de la retroalimentación proporcionada por los alumnos.

- Publicar todo el conjunto de videos docentes desarrollados en un curso abierto dentro de la plataforma OCW de la Universidad de Zaragoza, como apoyo el estudio de los fundamentos básicos de la termodinámica y la ingeniería térmica

- Consolidar y ampliar un grupo de profesores especializados en la elaboración y utilización de vídeos docentes como objetos de aprendizaje conceptuales, procedimentales y actitudinales en el ámbito de la termodinámica y la ingeniería térmica.

(c) EY-NC-ND 2017, Universitat Politècnica de València 


\section{Objetivos}

El objetivo general de este trabajo es presentar las actividades realizadas en un proyecto de innovación orientado a la elaboración de un conjunto de 25 nuevos objetos de aprendizaje modulares en forma de vídeos docentes con licencias Creative Commons que faciliten el aprendizaje autónomo del alumnado en el campo de la termodinámica y la ingeniería térmica.

Se persigue divulgar una metodología, aplicable a cualquier área de conocimiento, que permita la definición y desarrollo de vídeos docentes, así como la evaluación de las mejoras obtenidas en el aprendizaje mediante su uso en asignaturas de grados universitarios. Asimismo, a través del análisis de los resultados obtenidos, se pretende demostrar que el uso de vídeos docentes permite mejorar la eficiencia en el proceso de consecución por parte del alumnado de los resultados y las competencias específicas de las asignaturas dentro de este campo, mejorando así los resultados de la evaluación y el grado de motivación y satisfacción de estudiantes y profesores.

\section{Desarrollo de la innovación}

La Tabla 1 resume las principales actividades que forman parte de la metodología seguida en este proyecto de innovación docente durante el año académico 2016-17 para el desarrollo de videos docentes y su integración en un OCW. En cuanto al calendario de dichas actividades, las tres primeras tuvieron lugar al inicio del curso académico, las actividades 4 y 5 se están desarrollando durante el curso y, finalmente, la actividad 6 se realizará al final del curso académico, tras la correspondiente validación y autorización por parte de la Universidad de Zaragoza.

Tabla 1. Metodología seguida en el proyecto de innovación docente durante el curso 2016-17

\begin{tabular}{ll}
\hline $\mathbf{N}^{\mathbf{o}}$ & \multicolumn{1}{c}{ Actividad } \\
\hline 1 & Actualización y mejora de los vídeos docentes del curso anterior \\
\hline 2 & Planificación de los nuevos vídeos docentes a realizar \\
\hline 3 & Preparación de cuestionarios para la evaluación de la mejora del proceso de enseñanza-aprendizaje \\
\hline 4 & Creación y uso de los vídeos docentes y lanzamiento de los cuestionarios \\
\hline 5 & Recopilación y análisis de datos. Planteamiento de acciones de mejora \\
\hline 6 & Publicación de la colección completa de vídeos docentes en una plataforma OCW \\
\hline
\end{tabular}

A partir del análisis de los vídeos docentes elaborados en el curso anterior, realizado a partir del feedback proporcionado por los estudiantes, se plantea en primer lugar, la reedición parcial de los vídeos elaborados con objeto de corregir algunas deficiencias detectadas e incorporar diversas mejoras. De este modo, se consigue la mejora continua del trabajo realizado.

El siguiente paso consiste en la planificación de los vídeos/objetos de aprendizaje a realizar, su temática y duración, la definición de las asignaturas en las que se van a utilizar, el 
profesor responsable de su elaboración, así como la fecha prevista de realización. En este punto se incluye también la definición de una plantilla común para todos los videos y la selección de las licencias Creative Commons (CC) para los vídeos elaborados. En la Tabla 2 se muestra la temática del conjunto completo de los 25 videos docentes -nuevos o existentes- que se integrarán en el curso $\mathrm{OCW}$, indicándose entre paréntesis el número total de vídeos por cada tema.

Tabla 2. Temática (y número) de vídeos docentes utilizados en las asignaturas

\begin{tabular}{ll}
\hline \multicolumn{2}{c}{ Temas } \\
\hline Tutorial del programa “Engineering Equation Solver”-EES (3) & Ciclos de refrigeración (2) \\
\hline Propiedades de las sustancias puras (4) & Psicrometría (2) \\
\hline Modelo de gas ideal (2) & Método de diferencias finitas-MDF (2) \\
\hline Balance de energía en sistemas cerrados (1) & Intercambiadores de calor (2) \\
\hline Balance de masa y energía en volúmenes de control (2) & Convección forzada exterior (1) \\
\hline Ciclos de potencia con turbina de gas-Brayton (1) & Motores Térmicos (1) \\
\hline Ciclos de potencia con turbina de gas-Rankine (2) & \\
\hline
\end{tabular}

Tal como se puede observar en la Tabla 3, las cinco asignaturas seleccionadas para el uso de los vídeos docentes se imparten en tres titulaciones distintas de la Universidad de Zaragoza

Tabla 3. Asignaturas seleccionadas para el uso de vídeos docentes

\begin{tabular}{llcccc}
\hline \multicolumn{1}{c}{ Asignatura } & \multicolumn{1}{c}{ Titulación } & Curso & $\begin{array}{c}\text { Créditos } \\
\text { ECTS }\end{array}$ & $\begin{array}{c}\mathbf{N}^{\mathbf{0}} \mathbf{d e} \\
\text { vídeos } \\
\text { usados }\end{array}$ & $\begin{array}{c}\mathbf{N}^{\mathbf{0}} \text { de } \\
\text { estudiantes }\end{array}$ \\
\hline $\begin{array}{l}\text { Termodinámica Técnica y } \\
\text { Fundamentos de Transmisión } \\
\text { de Calor }\end{array}$ & $\begin{array}{l}\text { Grado en Ingeniería } \\
\text { de Tecnologías } \\
\text { Industriales }\end{array}$ & $2^{\text {o }}$ & 6 & 19 & 70 \\
\hline $\begin{array}{l}\text { Termodinámica Técnica y } \\
\text { Fundamentos de Transmisión } \\
\text { de Calor }\end{array}$ & $\begin{array}{l}\text { Grado en Ingeniería } \\
\text { Mecánica }\end{array}$ & $2^{\text {o }}$ & 6 & 19 & 64 \\
\hline $\begin{array}{l}\text { Termodinámica Técnica y } \\
\text { Fundamentos de Transmisión } \\
\text { de Calor }\end{array}$ & $\begin{array}{l}\text { Grado en Ingeniería } \\
\text { Electrónica y } \\
\text { Automática }\end{array}$ & $2^{\text {o }}$ & 6 & 10 & 85 \\
\hline Ingeniería Térmica & $\begin{array}{l}\text { Grado en Ingeniería } \\
\text { de Tecnologías } \\
\text { Industriales }\end{array}$ & $2^{\text {o }}$ & 6 & 2 & 50 \\
\hline Máquinas y Motores Térmicos & $\begin{array}{l}\text { Grado en Ingeniería } \\
\text { Mecánica }\end{array}$ & $3^{\circ}$ & 6 & 2 & 70 \\
\hline
\end{tabular}


La siguiente actividad es la preparación de cuestionarios para evaluar cuantitativamente el grado de satisfacción y la mejora del proceso de enseñanza-aprendizaje obtenida a través de los vídeos docentes. Se plantean tres cuestionarios anónimos a realizar en distintos momentos del curso: un cuestionario inicial, donde se pregunta al alumnado sobre sus expectativas en cuanto a su participación en el proyecto de innovación docente; un cuestionario intermedio, donde el alumnado hace una valoración de cada video docente visionado en el contexto de la asignatura en la que se utiliza; y un cuestionario final, donde el alumnado hace una valoración final del proyecto de innovación docente. Tanto el cuestionario intermedio como el cuestionario final están realizados en base a una escala de tipo Likert de 5 puntos ( 1 a 5 ).

Tras el lanzamiento de los cuestionarios y videos docentes en la plataforma Moodle de la Universidad de Zaragoza, se recopilan y analizan todas las respuestas a los cuestionarios con objeto de poder implementar acciones de mejora en los videos, corregir posibles deficiencias detectadas y definir nuevas necesidades docentes para próximos cursos.

El conjunto completo de 25 vídeos realizados serán publicados al final del curso académico dentro de la plataforma OCW de la Universidad de Zaragoza (http://ocw.unizar.es/ocw). Para ello se creará un curso OCW denominado "Material audiovisual de apoyo al estudio de la termodinámica y la ingeniería térmica" en el que se integrarán de un modo coherente todos los vídeos realizados. Esta plataforma OCW de carácter abierto será la utilizada para la visualización de los vídeos docentes en próximos cursos académicos en sustitución de la plataforma interna Moodle. De este modo, cualquier estudiante o persona interesada en esta materia podrá acceder libremente a todos los objetos de aprendizaje creados.

\section{Resultados}

Puesto que los resultados de la aplicación de los vídeos docentes en las cinco asignaturas mostradas en la Tabla 3 han sido similares, seguidamente, a modo de ejemplo, se presentan los principales resultados obtenidos en una asignatura. En concreto, se ha seleccionado la asignatura "Termodinámica Técnica y Fundamentos de Transmisión de Calor", ya que cuenta con un mayor número de estudiantes participantes (70), además de ser la asignatura en la que se ha utilizado un mayor número de vídeos (19). Esta asignatura se imparte en el segundo año del Grado en Ingeniería de Tecnologías Industriales. La asignatura consta de 6 créditos ECTS, equivalentes a 150 horas de trabajo, que incluyen 60 horas presenciales repartidas entre clases magistrales, resolución de problemas y prácticas de laboratorio/simulación, y 90 horas no presenciales dedicadas al estudio personal, la resolución de trabajos tutorados y la evaluación.

La participación de los estudiantes en los distintos cuestionarios planteados ha disminuido a lo largo del curso, manteniéndose no obstante elevada en aquellas encuestas marcadas como obligatorias. Teniendo en cuenta lo anterior, la participación alcanzó el 97\% en el cuestionario inicial, el $52 \%$ en el cuestionario intermedio, descendiendo hasta el $15 \%$ en el cuestionario final, debido a que éste fue realizado en fechas próximas al periodo de exámenes. 


\subsection{Cuestionario inicial}

Casi el $20 \%$ de los estudiantes encuestados manifiesta que ha visualizado previamente distintos tipos de videos docentes actualmente proporcionados en un total de diez asignaturas pertenecientes al Grado en Ingeniería de Tecnologías Industriales. Sin embargo, la mayoría de los estudiantes (78\%) consideran insuficiente el uso actual de estos elementos de aprendizaje, afirmando que suelen buscar y visualizar por su cuenta vídeos docentes disponibles en la red relacionados con las temáticas de las asignaturas de su titulación. Por tanto, se aprecia un destacado interés hacia el uso de videos docentes para la mejora de los resultados de aprendizaje.

Por otra parte, entre los diferentes tipos de videos docentes que se pueden plantear, la mayoría de los estudiantes afirman que están interesados en visualizar videos de aplicaciones prácticas relacionadas con la asignatura $(29 \%)$ y en vídeos específicamente realizados por el profesor para explicar los contenidos de la asignatura (25\%). El resto de estudiantes, muestra su preferencia por visualizar vídeos ya existentes recomendados por el profesor, relativos a salidas profesionales $(16 \%)$, noticias $(15 \%)$ y conferencias $(15 \%)$ relacionadas con la temática de la asignatura.

\subsection{Cuestionario intermedio}

Para cada video docente elaborado se planteó un cuestionario según una escala de tipo Likert de 5 puntos ( 1 a 5). En la Fig. 1 se presentan los valores estadísticos promedio de los distintos aspectos evaluados en los videos docentes usados. Se trata, por tanto, de los valores promedio obtenidos para el conjunto de 19 vídeos utilizados en la asignatura seleccionada, no habiéndose detectado variaciones significativas en la valoración individual de cada vídeo docente utilizado. Debe destacarse que la mayoría de los estudiantes (más del $40 \%$ ) otorga una puntuación de 4 puntos en los aspectos de amenidad, duración, utilidad para la resolución de dudas y calidad técnica de los videos docentes. Asimismo el 50\% de los estudiantes asigna una puntuación de 4 puntos como calificación global de los videos. Por tanto, se concluye que la mayoría de los estudiantes han quedado muy satisfechos con los videos visualizados. 


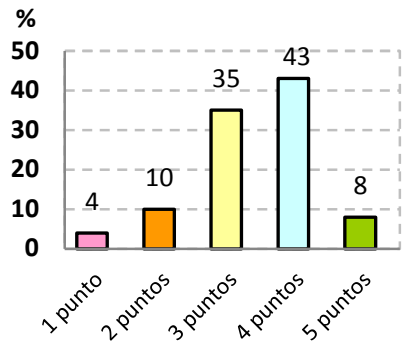

Amenidad y capacidad de motivación
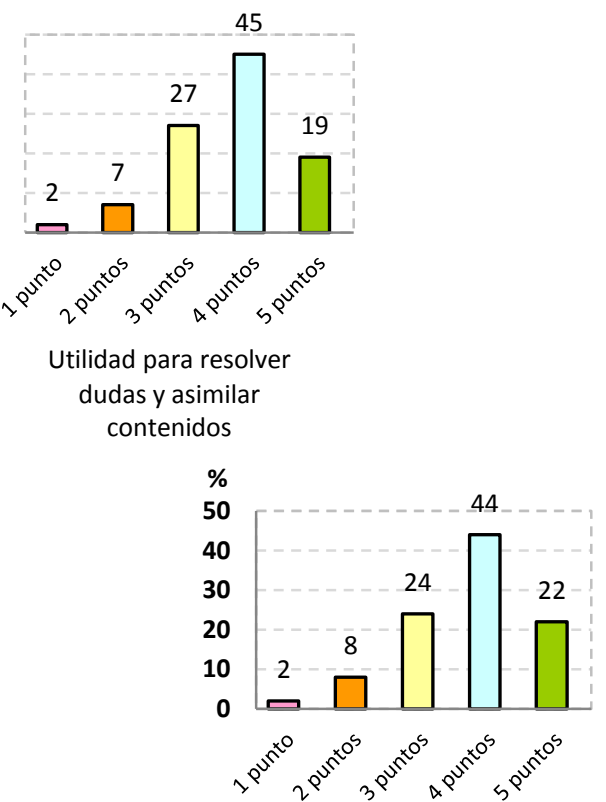

Calidad técnica del vídeo

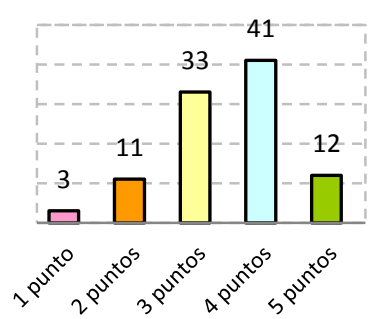

Adecuación de la duración del vídeo

Fig. 1 Valores estadísticos promedio de la evaluación de los vídeos docentes en la asignatura objeto de análisis

\subsection{Cuestionario final}

De cara a la valoración final se planteó igualmente un cuestionario con una escala de tipo Likert de 5 puntos ( $1=$ nada de acuerdo, $2=$ poco de acuerdo, $3=$ algo de acuerdo, 4=bastante de acuerdo, $5=$ totalmente de acuerdo), cuyos resultados se muestran en la Fig. 2.

Alrededor del 50\% de los estudiantes están en desacuerdo o muy en desacuerdo con el hecho de que visualizar videos les haya restado tiempo de estudio. Por otra parte, aunque la mitad de los estudiantes piensa que ha mejorado su proceso de aprendizaje, el mismo porcentaje considera que no hay una relación clara entre la visualización de videos y el aumento de su motivación para el estudio. Esto podría explicarse por la complejidad y variedad de factores que influyen en la motivación de los estudiantes. Por otro lado, el $60 \%$ de los estudiantes considera deseable o altamente deseable extender el uso de videos docentes a otras asignaturas de su titulación. Su interés en disponer de más videos docentes 
para la asignatura analizada no es tan claro, tal vez debido al alto número de videos utilizados a lo largo del curso. Por último, cabe destacar que todos los estudiantes dan una calificación global al proyecto de innovación docente superior a 3 puntos, y el $60 \%$ otorga una puntuación entre 4 y 5 puntos.

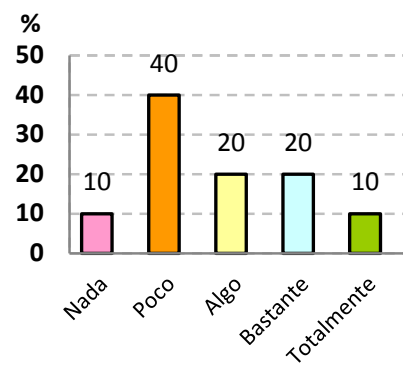

Visionar vídeos me ha quitado tiempo de estudio

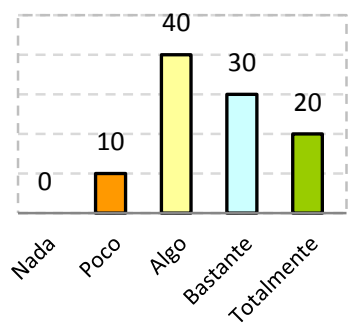

Visionar videos ha mejorado mi aprendizaje

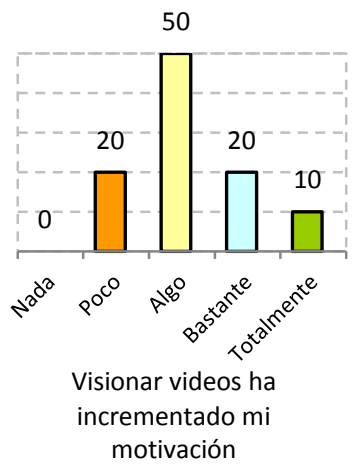

motivación 


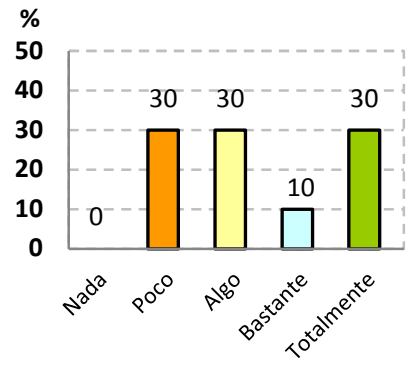

Conveniencia de disponer de más vídeos docentes

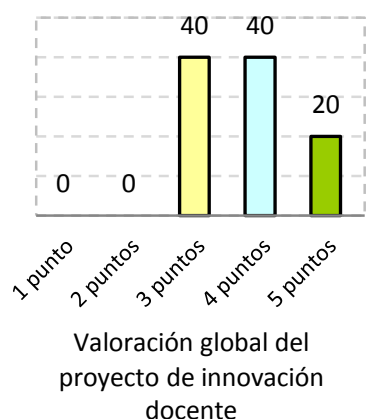

Fig. 2 Valores obtenidos del cuestionario final en la asignatura objeto de análisis

\footnotetext{
Valoración global del

royecto de innovación
}

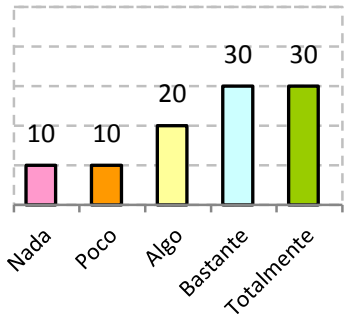

Conveniencia de usar vídeos docentes en otras asignaturas 


\section{Conclusiones}

Aunque el proyecto de innovación docente presentado en este trabajo está siendo aplicado en el ámbito de la termodinámica y la ingeniería térmica, la metodología seguida para el desarrollo de videos docentes y la evaluación cuantitativa de su impacto en el proceso de enseñanza-aprendizaje es completamente replicable en cualquier disciplina y área de conocimiento. Además, la metodología es relativamente fácil de implementar y no requiere de grandes esfuerzos adicionales por parte de los profesores, siendo especialmente adecuada para redes colaborativas de profesores interesados en la innovación y la mejora de la actividad docente. Los resultados presentados en esta comunicación demuestran que la visualización de videos docentes en un contexto universitario permite mejorar el proceso de aprendizaje, aprovechando mejor el tiempo dedicado a las clases magistrales y las sesiones prácticas.

\section{Referencias}

REHAK, D. y MASON, R. (2003). "Engaging with the Learning Object Economy", en Reusing Online Resources: A Sustainable Approach to E-Learning (A. Littlejohn, ed.), pp. 22-30, London: Kogan Page.

WINDLE, R.J. et al. (2011). "The characteristics of reusable learning objects that enhance learning" en British Journal of Educational Technology, vol. 42, no.5, pp. 811-823.

ZABALZA, I., PEÑA, B., LLERA, E.M., MARTíNEZ, A. y ROMEO, L.M. (2017). "Development of educational videos as reusable learning objects for their integration into an Open Courseware on fundamentals of thermodynamics and thermal engineering" en Proceedings of the 11th annual International Technology, Education and Development Conference (INTED 17). Valencia.

PEÑA, B., ZABALZA, I., USÓN, S., LLERA, E.M., MARTÍNEZ, A. y ROMEO, L.M. (2017). "Pilot experience for the application of the flipped classroom in subjects of the field of thermal engineering" en Proceedings of the 11th annual International Technology, Education and Development Conference (INTED 17). Valencia.

KAY, R.H. y KNAACK, L. (2007). "Evaluating the learning in learning objects" en Open Learning, vol. 22, no.1, pp. 5-28.

LEACOCK, T.L. y NESBIT, J.C. (2007). "A Framework for Evaluating the Quality of Multimedia Learning Resources" en Educational Technology \& Society, vol. 10, no. 2, pp. 44-59.

SINGH, R:G. y BERNARD, M.A. (2016). "Quality assurance for reusable learning objects on a peerto-peer network" en International Journal of Emerging Technologies in Learning, vol. 11, no. 10, pp. $4-10$.

PINTO, M., GOMEZ-CAMARERO, C. y FERNÁNDEZ-RAMOS, A. (2012). "Los recursos educativos electrónicos: perspectivas y herramientas de evaluación" en Perspect. em Ciência da Informação, vol. 17, no.3, pp. 82-99. 